\title{
Levosimendan protects from sepsis-inducing cardiac dysfunction by suppressing inflammation, oxidative stress and regulating cardiac mitophagy via the PINK-1-Parkin pathway in mice
}

\author{
Jian Shi ${ }^{1}$, Yuhong Chen ${ }^{1,2}$, Haijun $\mathrm{Zhi}^{3}$, Hui An ${ }^{4}$, Zhenjie $\mathrm{Hu}^{1,2}$ \\ ${ }^{1}$ Department of Intensive Care Unit, Hebei Medical University Fourth Affiliated Hospital and Hebei Provincial Tumor Hospital, Shijiazhuang, \\ China; ${ }^{2}$ Hebei Key Laboratory of Critical Disease Mechanism and Intervention, Shijiazhuang, China; ${ }^{3}$ Emergency Department, Cangzhou Central \\ Hospital, Cangzhou, China; ${ }^{4}$ Department of Intensive Care Unit, Baoding First Central Hospital, Baoding, China \\ Contributions: (I) Conception and design: Z Hu, Y Chen, J Shi; (II) Administrative support: Z Hu; (III) Provision of study materials or patients: Z Hu, \\ Y Chen, J Shi, H Zhi; (IV) Collection and assembly of data: J Shi, H Zhi; (V) Data analysis and interpretation: J Shi; (VI) Manuscript writing: All \\ authors; (VII) Final approval of manuscript: All authors. \\ Correspondence to: Zhenjie Hu. Department of Intensive Care Unit, Hebei Medical University Fourth Affiliated Hospital and Hebei Provincial Tumor \\ Hospital, 12 Jiankang Road, Shijiazhuang 050011, China. Email: syicuhzj@163.com.
}

Background: Sepsis is a leading cause of death in China, the mortality rate of which is elevated when cardiac dysfunction is induced. Levosimendan is used for the treatment of decompensated cardiac failure. In this study, we sought to investigate the role of levosimendan in the inflammation, oxidative stress, and mitophagic response of the septic heart.

Methods: A lipopolysaccharide (LPS)-induced septic myocardial dysfunction mouse model was established. To study the relationship between levosimendan and inflammation, oxidative stress, and mitophagy response, mice were pretreated with mdivi-1 (an inhibitor of mitophagy) prior to LPS administration. Levosimendan was given $(24 \mu \mathrm{g} / \mathrm{kg})$ via intraperitoneal injection $3 \mathrm{~h}$ after LPS had been administered. At $6 \mathrm{~h}$ after LPS injection, echocardiographic analysis, enzyme-linked immunosorbent assay (ELISA), oxidative stress index, myocardial pathological changes, transmission electron microscopy (TEM), immunofluorescence, and western blot were used to investigate the protective effects of levosimendan against LPS-induced myocardial dysfunction.

Results: In the sepsis model, levosimendan markedly ameliorated myocardial dysfunction, decreased the release of myocardial enzymes and inflammatory cytokines, improved oxidative stress index and myocardial pathological changes, reduced mitochondrial division, and activated mitophagy. To confirm whether the protection of levosimendan was mediated by mitophagy, a mitophagy inhibitor-mdivi-1 was used in this study. It significantly impaired the protective effects of levosimendan. In addition, our studies further confirmed the protection of levosimendan against LPS-induced myocardial injury and the mechanisms involving PINK-1-Parkin mediated mitophagy signaling.

Conclusions: Levosimendan was able to rescue the LPS-induced cardiac dysfunction mice, supporting its mechanism of action by suppressing inflammation, oxidative stress, and directly targeting the PINK-1Parkin pathway.

Keywords: Levosimendan; lipopolysaccharide (LPS); septic cardiac dysfunction; mitochondrial dynamics; mitophagy

Submitted Dec 31, 2021. Accepted for publication Feb 16, 2022.

doi: $10.21037 / \mathrm{atm}-22-483$

View this article at: https://dx.doi.org/10.21037/atm-22-483 


\section{Introduction}

Sepsis is a leading cause of death in China, the mortality rate of which is elevated when cardiac dysfunction is induced. About $46 \%$ of patients with sepsis have myocardial injury or cardiac dysfunction (1); however, the mechanism of septic cardiac dysfunction is not completely understood. To date, mitochondrial dysfunction has been confirmed as playing a pivotal role in sepsis-induced cardiac dysfunction (2). In vitro and in vivo studies have indicated that mitochondria structure and function is regulated by sepsis in myocardial volume $(3,4)$. The quality and quantity control of mitochondria is mainly the balance of mitochondrial dynamic function, that is, fission, fusion, mitophagy, and mitochondrial biological function (5).

Autophagy appears to be a double-edged sword, as under different degrees of stimulation the regulation of cell survival is inconsistent (6). Under normal physiological responses or mild stress, autophagy is appropriate and could promote survival. The process during which dysfunctional mitochondria is segregated and eliminated through autophagy is known as mitophagy (7). However, under severe stress, autophagy is inappropriate, resulting in massive self-degradation or accumulation of toxic substances; neither of these outcomes is adaptive. Evidence from a mouse LPS model showed that enhanced autophagy in severe sepsis protects the myocardium (8). The Pink-1/Parkin pathway induced by the phosphatase and PTEN, is the most representative signaling pathway in mitophagy. It was reported that inhibition of PINK1/ Parkin pathway protein expression can reduce the level of mitophagy, and aggravate mitochondrial injury, whereas activation of PINK1/Parkin pathway can play a protective effect on heart (9).

Levosimendan is used for the treatment of decompensated cardiac failure as a calcium sensitizer and mitochondrial potassium-adenosine triphosphate (ATP) channel opening agent $(10,11)$. But the application of levosimendan in sepsis-inducing cardiac dysfunction is still controversial. Some small clinical studies have confirmed Septic patients with myocardial dysfunction may partly benefit from levosimendan than dobutamine (12). Over the recent past, it has become evident that decompensated cardiac failure is associated with subclinical inflammation oxidative excess and inappropriate mitochondrial quality control $(13,14)$. Some animal studies have showed that besides levosimendan's positive inotropic effect, it has pleiotropic actions including antioxidant, anti-inflammatory, and anti-apoptosis effects (15-17). Grossini et al. indicated that levosimendan participates in autophagy during regional myocardial ischemia in pigs (10). Given that antiinflammatory, antioxidant, and anti-apoptosis properties are involved in cardioprotection, we sought to determine the role of levosimendan in the mitophagic response of the septic heart, so as to determine whether levosimendan can improve cardiac function in sepsis-inducing cardiac dysfunction through other ways. Establishment of a mouse sepsis myocardial injury model via LPS intraperitoneal injection is simple, economical, and similar to the clinical reality. It is an important method to study sepsis myocardial injury. We evaluated (I) the effect of levosimendan in sepsisinduced cardiac dysfunction; (II) the underlying molecular mechanisms; and (III) we sought to clarify the role of levosimendan in quality control of cardiac mitochondria through mitophagy. We present the following article in accordance with the ARRIVE reporting checklist (available at https://atm.amegroups.com/article/view/10.21037/atm$22-483 / \mathrm{rc})$.

\section{Methods}

\section{Animals and treatment}

Experiments were performed using male healthy C57BL/6J mice aged $8-10$ weeks and weighing $21 \pm 3.5 \mathrm{~g}$ (Vital River Laboratories, Beijing, China). Mice were maintained under standard pathogen-free conditions at about $22{ }^{\circ} \mathrm{C}$ with a 12-h light-dark cycle with free access to a standard rodent diet and water. Experiments were performed under a project license (No. 2020002) granted by the Laboratory Animal Ethical Committee Fourth Hospital Hebei Medical University, in compliance with the National Institutes of Health (USA) Guidelines for the care and use of animals (NIH Publication No. 85-23, revised 1985: http:// grants1.nih.gov/grants/olaw/references/phspol.htm). All experiments were performed in the Animal Experiment Center of the Fourth Hospital of Hebei Medical University.

The septic myocardial dysfunction model was established by intraperitoneal injection of LPS $(10 \mathrm{mg} / \mathrm{kg}$; Solarbio, Beijing, China) (18). The study comprised two parts. In the first part, mice were randomly assigned into control group ( $\mathrm{n}=6$ ), levosimendan (Levo) group $(\mathrm{n}=6)$, LPS group $(\mathrm{n}=6)$, and LPS + levo group $(\mathrm{n}=6)$ according to the random number method. Mice allocated to the LPS group and LPS + levo group underwent echocardiography (echo) at $3 \mathrm{~h}$ after LPS injection. If myocardial dysfunction was indicated 
by echo, they entered the experimental process; if not, they were euthanized by cervical dislocation. Levosimendan (Qilu Pharmaceutical Co., Ltd, Shandong, China) was injected via an intraperitoneal injection at $24 \mu \mathrm{g} / \mathrm{kg}$ (dissolved in Glu $0.5 \%$ ) according to previous research and preliminary experiment in $3 \mathrm{~h}$ after the LPS intervention (19). In the second part, mice were randomly assigned into a control group $(n=6)$, LPS group $(n=6)$, LPS + levosimendan group ( $\mathrm{n}=6)$, and LPS + levo + Mdivi-1 group ( $\mathrm{n}=6)$. Mice assigned into the LPS group, LPS + levo, and LPS + levo+Mdivi-1 group underwent echo at $3 \mathrm{~h}$ after LPS. If myocardial dysfunction was indicated by echo, they entered the experimental process; if not, they were euthanized by cervical dislocation. As an activity inhibitor of mitophagy, Mdivi-1 (dissolved in dimethyl sulfoxide (DMSO), 338967-87-6, MCE, Monmouth Junction, NJ, USA) was administrated $1 \mathrm{~h}$ prior to the LPS injection at a dose of $25 \mathrm{mg} / \mathrm{kg}$ intraperitoneally (20). Mice were euthanized by cervical dislocation after echocardiographic experiments, and the blood and myocardial tissues were collected. Blood samples were immediately centrifuged (3,000 rpm, $15 \mathrm{~min}$ ) at $4{ }^{\circ} \mathrm{C}$ to isolate serum. The serum was then stored at $-80{ }^{\circ} \mathrm{C}$ until used. The myocardial tissues were washed in phosphate-buffered saline (PBS), and stored at $-80^{\circ} \mathrm{C}$.

\section{Echo}

When mice were anesthetized with isoflurane (2\%), echocardiographic examinations were performed by an ultrasound system (Vevo 2100 imaging system, VisualSonics, Ontario, Canada) equipped with a $13-\mathrm{MHz}$ linear transducer and professional ultrasound technicians who were blind to animal grouping. The left ventricular ejection fraction (LVEF) and left ventricular fractional shortening (LVFS) were calculated from M-mode images of left ventricular (LV) dimensions.

\section{Heart biological markers of myocardial injury and inflammation}

Heart biological markers of myocardial injury cardiac troponin I (cTnI) (Cat No. E-EL-M1203c, Elabscience biotechnology, Wuhan, China), Creatine kinasemyocardial isoenzyme (CK-MB) (Cat No. E-EL-M0355c, Elabscience Biotechnology, Wuhan, China), and markers of inflammation tumor necrosis factor- $\alpha(\mathrm{TNF}-\alpha)$ (Cat No. KE10002, Proteintech, Wuhan, China), interleukin-1 $\beta$ (IL1ß) (Cat No. KE10003, Proteintech, Wuhan, China) levels were detected using enzyme-linked immunosorbent assay (ELISA) kits.

\section{Heart oxidative stress indicators}

We collected the myocardial tissues of mice as described above. The CuZn-superoxide dismutase (CuZn-SOD), glutathione-peroxidase (GSH-Px), and malondialdehyde (MDA) levels were detected using biochemical analysis kits (Elabscience biotechnology, Wuhan, Hubei, China) according to the manufacturers' instructions.

\section{Transmission electron microscopy (TEM)}

Myocardial tissues of the $\mathrm{LV}$ wall were cut into $1 \mathrm{~mm}^{3}$ thick slices and fixed (buffer: $1 \% \mathrm{OsO}_{4}$ in $0.1 \mathrm{M} \mathrm{PB}, \mathrm{pH}$ 7.4), embedded, and polymerized. Sections $(60-80 \mathrm{~nm})$ were cut, stained, and examined under a transmission electron microscopy (TEM; HT7800/HT7700, Hitachi Ltd., Tokyo, Japan). The autophagosome was observed and counted. We selected 5 random regions for each sample for quantification (21).

\section{Histological analysis}

Myocardial tissues were fixed in $4 \%$ paraformaldehyde at room temperature for $48 \mathrm{~h}$, paraffin-embedded, sectioned, and then hematoxylin-eosin ( $\mathrm{H} \& \mathrm{E})$ stained for morphological analysis. Slides were observed under a light microscope (DM3000 LED, Leica, Wetzlar, Germany). The changes of myocardial histological staining were scored as previous study described (22). The sections were graded (0 to $4+)$ for cellular infiltration and myocardial necrosis by two experienced observers who were blind to the experimental treatments. The grades were as follows: 0 , no myocardial lesion; 1 , lesions involving less than $25 \%$ of the myocardium; 2 , lesions involving $25-50 \%$ of the myocardium; 3 , lesions involving $50-75 \%$ of the myocardium; and 4 , lesions involving more than $75 \%$ of the myocardium. The score was calculated as the mean derived from two sections of each sample.

\section{Immunofluorescent microscopy}

After deparaffinization, the sections were incubated with a microtubule-associated protein light chain 3 (LC3) antibody (1:200, ab192890, Abcam, Cambridge, UK). We used LC3conjugated goat polyclonal secondary antibody to rabbit 
immunoglobulin $\mathrm{G}(\mathrm{IgG})-\mathrm{H} \& \mathrm{~L}$ as a secondary antibody (1:500, ab150081, Abcam, UK). These sections were then counterstained with Beyotime c1006 (Beyotime, Nanjing, china) and examined under the microscope (DM3000 LED, Leica, Germany).

\section{Western blot analysis}

Whole proteins were extracted from myocardial tissues in radioimmunoprecipitation assay (RIPA) buffer containing phosphatase inhibitor on ice, and quantified using a bicinchoninic acid (BCA) Kit (Cat No. E-BC-K318-M, Elabscience Biotechnology, Wuhan, China). After electrophoresis on $8 \%$ or $12 \%$ sodium dodecyl sulfate polyacrylamide gel electrophoresis (SDS-PAGE) gels, the proteins were blotted to polyvinylidene fluoride (PVDF) membranes (Millipore-Upstate, Beijing, China, 0.45 um). After blocking in 5\% skim milk (Cat No. 1172GR500, Saiguo, Guangzhou, China) or albumin (Solarbio, Beijing, China) in Tris-buffered solution containing $0.1 \%$ Tween-20 (TBST) for $2 \mathrm{~h}$ at $37^{\circ} \mathrm{C}$, the membranes were incubated at $4{ }^{\circ} \mathrm{C}$ for at least $16 \mathrm{~h}$ with primary antibodies against uncoupling protein 2 (UCP2; 1:1,000, Cat No. 11081-1AP, Proteintech, Wuhan, China), dynamin-related peptide 1 (Drp-1; 1:8000, Cat No. A2586, ABclonal, Wuhan, China), pDrp-1 (Ser616; 1:3,000, Cat No. \#12749, Signalway Antibody (SAB), College Park, MD, USA), tensin homolog (PTEN)-induced putative kinase 1 (PINK1; 1:1,000, Cat No. ab186303, Abcam, UK), Parkin (1:2,000, Cat No. ab77924, Abcam, UK), LC3 (1:3,000, Cat No. 14600-1AP, Proteintech, Wuhan, China), and beta-actin (1:50,000, catalog number AC026, ABclonal, Wuhan, China). After washing 3 times with TBST $(15 \mathrm{~min} \times 3)$, the membranes were incubated with secondary antibodies $(1: 10,000$, Cat No. SA00001-2, Proteintech, Wuhan, China) at room temperature for $2 \mathrm{~h}$. The protein bands were developed using an ECL detection kit and visualized using a BLT GelView 6000Plus (Bolu, Guangzhou, China). The band intensities were quantified using the AlphaView software (ProteinSimple, San Jose, CA, USA).

\section{Statistical analyses}

Statistical analysis was performed with SPSS software version 26.0 (IBM Corp., Chicago, IL, USA). Western blotting and immunofluorescent microscopy analysis were completed by scanning and analyzing the intensity of signals on film by using NIH Image J (https://imagej.nih.gov/ij/). Data were presented as the mean \pm standard deviation (SD). A one-way analysis of variance (ANOVA) following the least significant difference (LSD) or Student-Neuman-Keuls (SNK)-q test was carried out for comparisons of more than two groups. The $t$-test was used for comparisons between two groups. Statistical significance was defined as $\mathrm{P}<0.05$.

\section{Results}

Effects of levosimendan treatment on LPS-induced cardiac dysfunction mice

A total of 36 mice were obtained for this investigation, and 24 were included and completed. We excluded 12 mice because of no occurrence of myocardial dysfunction. Clinical signs of endotoxemia were observed in mice treated with LPS, including lethargy, mild closure of the eyes, ruffled fur, and diarrhea. No death was observed across all groups at $6 \mathrm{~h}$ after LPS injection and there was no significant difference in body weight among the groups. Compared to the control group, echo examinations revealed decreased LVEF and LVFS after LPS injection. Treatment of levosimendan could reverse cardiac dysfunction induced by LPS as indicated by a higher LVEF and LVFS (Figure 1A-1C). Furthermore, after LPS exposure, cTNI and CK-MB levels in serum expression were increased, and these effects were attenuated after levosimendan administration (Figure 1D,1E).

\section{Effects of levosimendan treatment on cardiac inflammation, oxidative stress, and pathological changes of mice with LPS-induced cardiac dysfunction}

The plasma TNF- $\alpha$ and IL- $1 \beta$ levels were significantly higher in the LPS group than in the other three groups. Treatment with levosimendan significantly reduced TNF- $\alpha$ and IL-1 $\beta$ levels (Figure 2A,2B).

Furthermore, compared to control mice, cardiac GSH-Px levels and $\mathrm{Cu} / \mathrm{ZnSOD}$ activities were decreased, but those of MDA were increased in LPS-treated mice. Levosimendan improved cardiac GSH-Px levels and $\mathrm{Cu} / \mathrm{ZnSOD}$ activities, and reduced cardiac MDA levels (Figure 2C-2E).

Additionally, levosimendan administration significantly ameliorated myocardial pathological changes caused by LPS (Figure $2 \mathrm{~F}-2 \mathrm{H}$ ). 


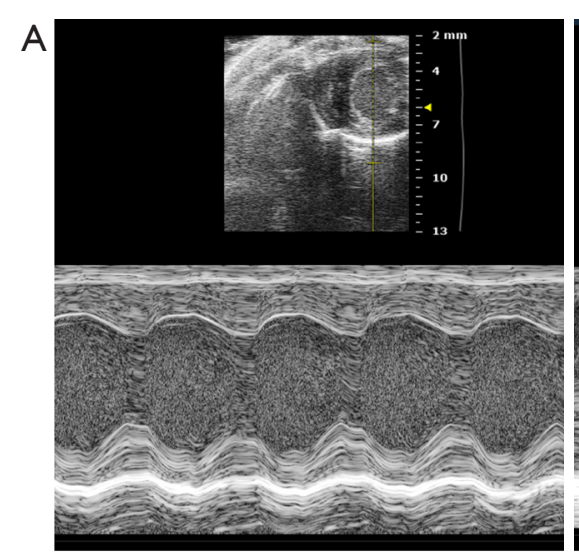

Control
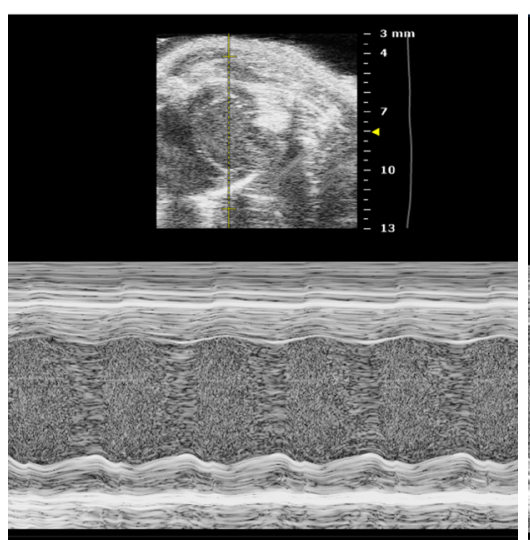

LPS

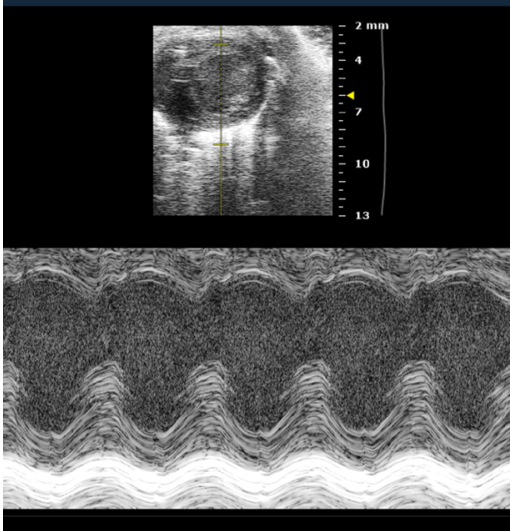

Levo
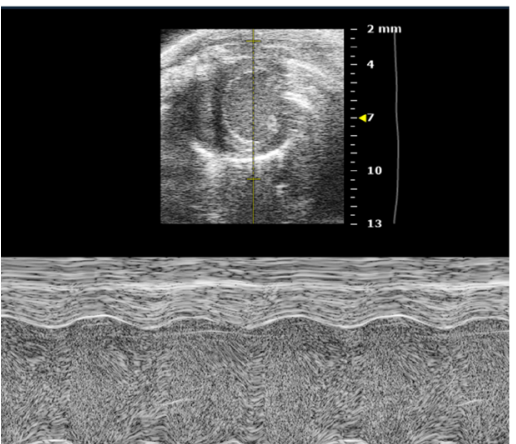

Q

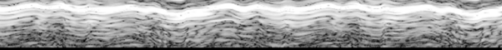
LPS + Levo
B

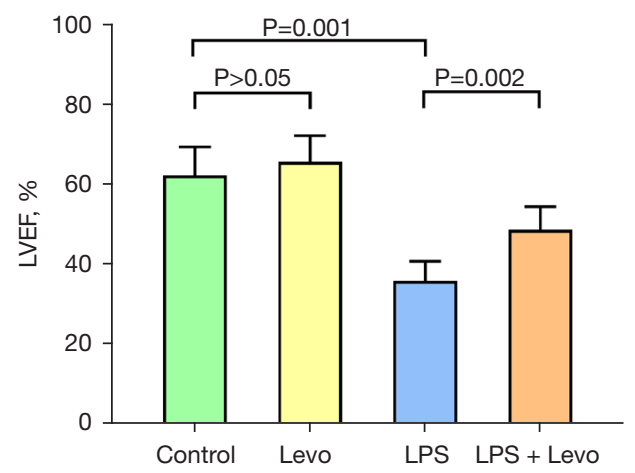

C

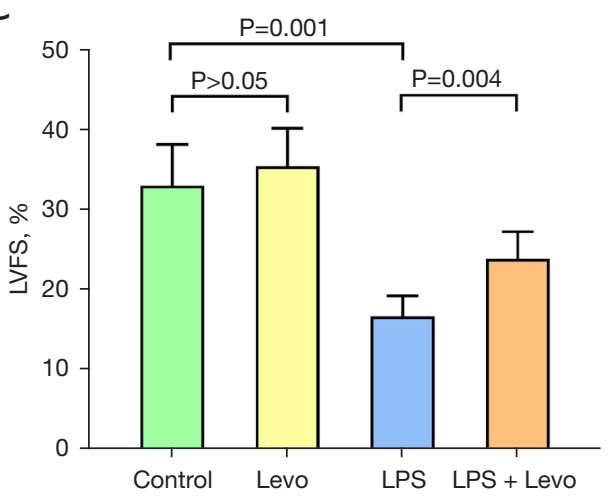

D

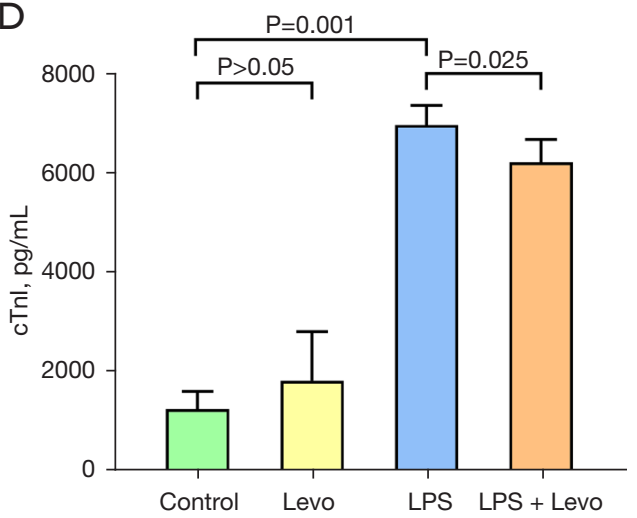

E

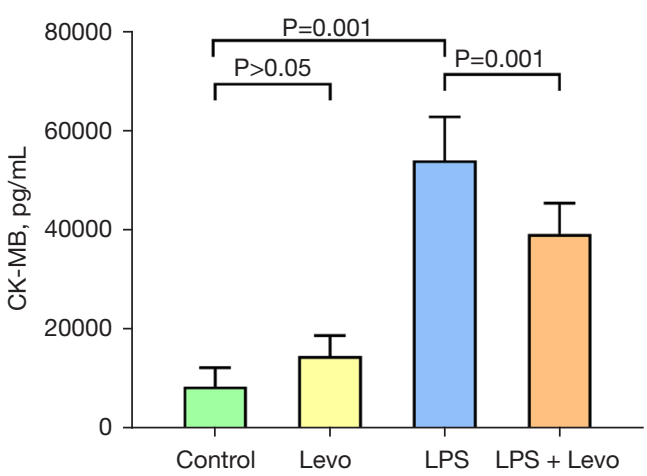

Figure 1 Levosimendan administration improves cardiac contractility and myocardial injury. (A) Representative M-mode images from mice in each group $(\mathrm{n}=6)$; $(\mathrm{B}, \mathrm{C})$ quantitative analysis of each group mice LVEF and LVFS (n=6); (D,E) evaluation of myocardial injury by cTnI and CK-MB in serum (n=6). LVEF, left ventricular ejection fraction; LVFS, left ventricular fractional shortening; cTnI, cardiac troponin I; CK-MB, creatine kinase-myocardial isoenzyme; LPS, lipopolysaccharide; Levo, Levosimendan. 

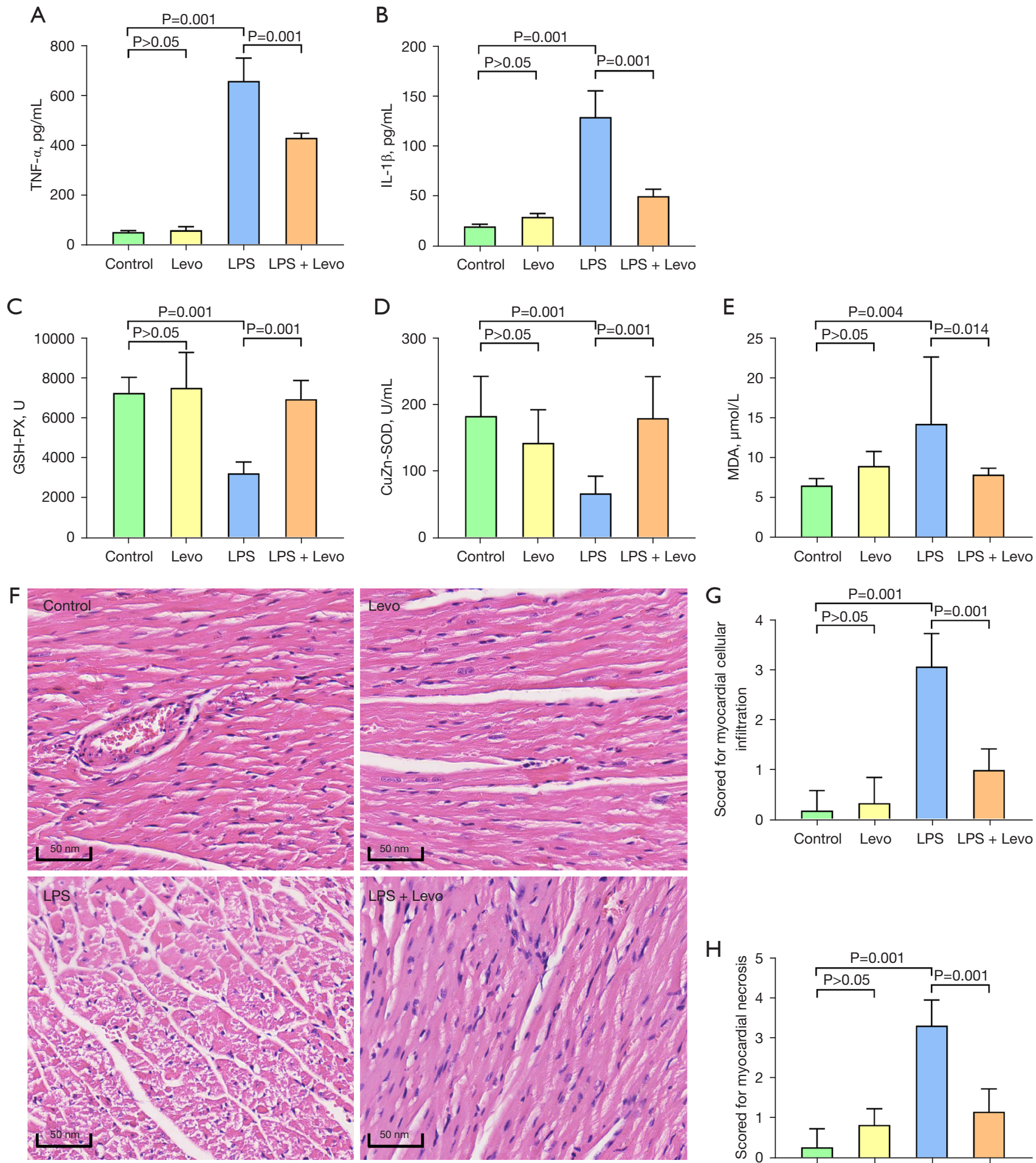

$\mathrm{H}$

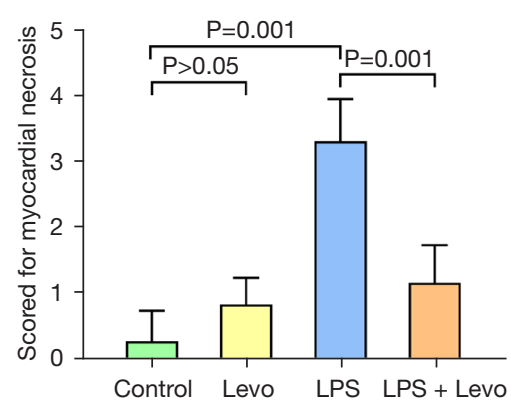

Figure 2 Levosimendan administration improves cardiac inflammation, oxidative stress and pathological changes. (A,B) Evaluation of cardiac inflammation by TNF- $\alpha$ and IL-1 $\beta$ in serum (n=6); (C-E) evaluation of cardiac oxidative stress by GSH-Px, CuZn-SOD, and MDA in serum $(n=6) ;(F)$ representative HE-stained cardiac sections $(n=6) ;(G, H)$ myocardial cellular infiltration and necrosis score of 4 groups $(\mathrm{n}=6)$. TNF- $\alpha$, tumor necrosis factor- $\alpha$; IL-1 $\beta$, interleukin-1 $\beta$; GSH-Px, glutathione-peroxidase; CuZn-SOD, CuZn-superoxide dismutase; MDA, malondialdehyde; HE, hematoxylin and eosin; LPS, lipopolysaccharide; Levo, Levosimendan. 


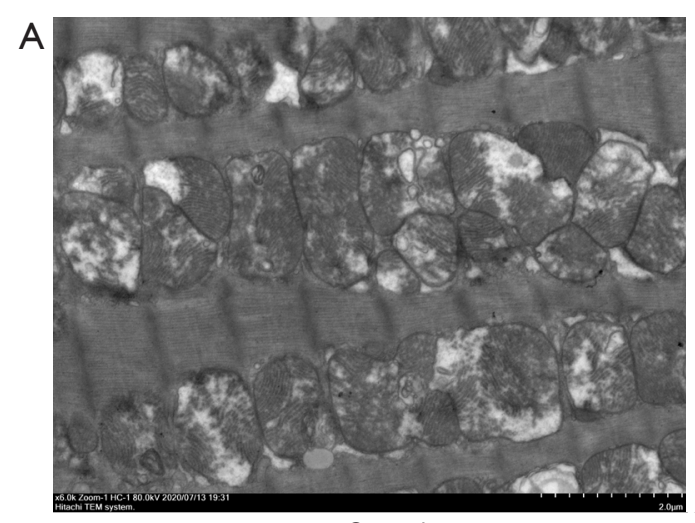

Control

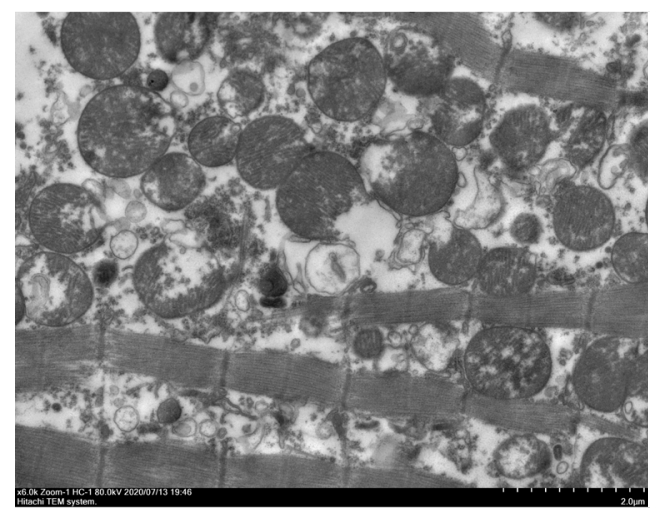

LPS

B

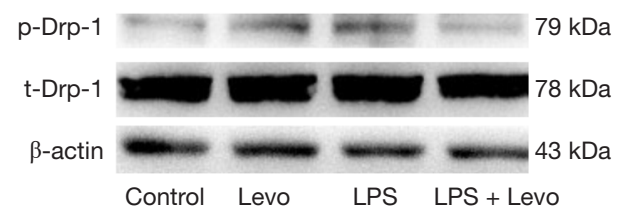

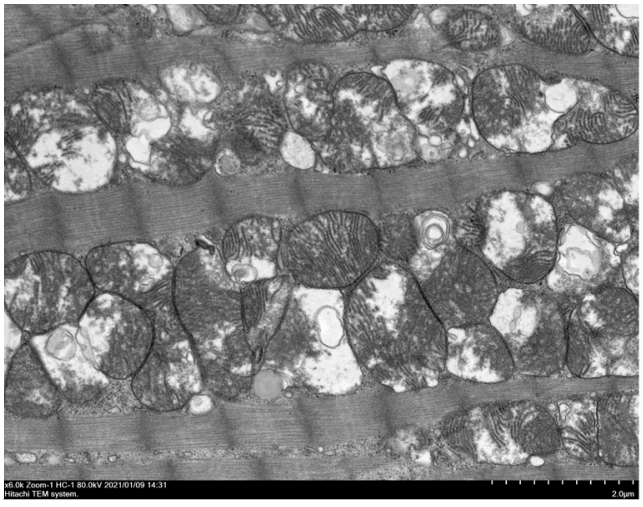

Levo

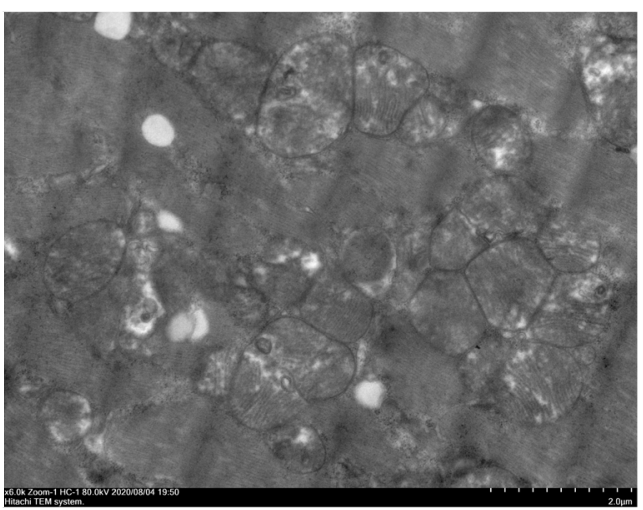

LPS + Levo

C

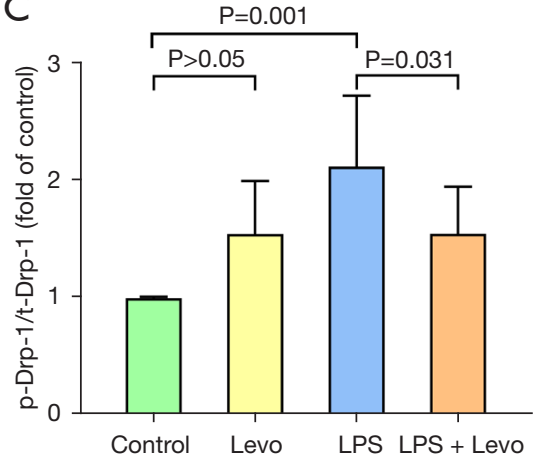

Figure 3 Levosimendan administration improves mitochondrial ultrastructure induced by LPS. (A) Representative images of mitochondrial morphology $(n=6)$; (B,C) representative bands of p-Drp-1 and Drp-1, and quantitative analysis of p-Drp-1 and Drp-1 expressions ( $\mathrm{n}=6$ ). LPS, lipopolysaccharide; Levo, Levosimendan.

\section{Effects of levosimendan on cardiac mitochondrial injury and fragmentation of mice with LPS-induced cardiac dysfunction}

Examination with TEM revealed normal mitochondrial structure in control group, including intact double membrane, dense matrix granules, and tightly packed cristae. The most obvious alterations induced by LPS were loss of internal cristae matrix granules and electrondense matrix and shift toward smaller cardiac mitochondria distribution. Levosimendan could prevent LPS-induced mitochondrial injury (Figure $3 A$ ).

In the LPS group, mitochondrial fragmentation was associated with phosphorylated Drp1 (phospho ser 616 Drp1) expression elevation, and levosimendan could 
prevented Drp1 phosphorylation (Figure 3B,3C).

\section{Effects of levosimendan on cardiac mitophagy of mice with LPS-induced cardiac dysfunction}

Conversion of the soluble form of LC3 (LC3-I) to autophagosome-associated and lipidated form (LC3-II) expression were used to assess mitophagy. The significant elevation in LC3-II to LC3-I ratio was observed in the LPS group, and levosimendan reinforced the LC3-II to LC3-I ratio in LPS group on LC3-positive punctate staining assessed by immunofluorescence (Figure $4 A, 4 B$ ) and western blot (Figure 4C,4D).

We found that LPS insult slightly increased PINK-1 and Parkin expression, which is a key pathway involved in the regulation of mitophagy, and levosimendan treatment further significantly increased PINK-1 and Parkin expression (Figure 4E, $4 F$ ).

\section{Mitophagy plays an important role in myocardial protection of levosimendan}

A total of 42 mice were utilized for this component and 24 were included and completed. We had to exclude 18 mice due to no occurrence of myocardial dysfunction. No death was observed across all groups at $6 \mathrm{~h}$ after LPS injection and there was no significant difference in body weight among the groups.

The critical role of mitophagy was further validated through PINK1-Parkin pathway in LPS-induced cardiac dysfunction. We investigated the effects of mitophagy inhibition by mdivi-1. Compared to the LPS + Levo group, administration of mdivi-1 significantly reduced LVEF and LVFS (Figure 5A-5C). In addition, mdivi-1 increased the levels of cTNI, CK-MB, TNF- $\alpha$, and IL- $1 \beta$ (Figure 5D-5G). Meanwhile, mdivi-1 was shown to reverse the improvement of oxidative stress index and myocardial pathological changes by levosimendan (Figure $5 \mathrm{H}-5 \mathrm{M}$ ).

Detection with TEM revealed that autophagosomes in the myocardium in the LPS group were significantly increased, compared to the Control group. Moreover, compared to the LPS group, levosimendan administration further increased the number of autophagosomes. Compared to the LPS + Levo group, the administration of mdivi-1 significantly decreased the number of autophagosomes (Figure 6A,6B). Immunofluorescence and western blot analysis showed that mdivi-1 significantly reduced LC3B-II/LC3B-I ratio (Figures $6 C, 6 D, 7 A, 7 B$ ). In addition, mdivi-1 could reduce the expression of PINK1 and Parkin but not affect the expression of p-DRP-1 (Figure $7 C-7 E)$.

\section{Discussion}

Sepsis is the leading cause of death in the intensive care unit (ICU), and sepsis-induced myocardial dysfunction is a major global healthcare problem. Sepsis induces myocardial mitochondrial structure damage and loss of mitochondrial function (23). In this study, we found that levosimendan significantly inhibited mitochondrial fission and strengthened myocardial mitophagy to reduce mitochondrial damage and improve cardiac function. Moreover, levosimendan treatment significantly activated PINK-1 and Parkin, and thus increased the autophagic LC3-II/LC3-I protein ratio. These data indicate that levosimendan regulates mitophagy activation and mitochondrial integrality preservation to improve cardiac function via PINK-1-Parkin mediated mechanisms.

Cardiac dysfunction is a critical component of multiorgan dysfunction in sepsis. It is manifested by the inhibition of systolic and diastolic cardiac function, elevation myocardial injury markers, and destruction of myocardial mechanisms (24). Our results suggested that LPS caused significant myocardial injury and cardiac function reduction represented by higher cTNI and CK$\mathrm{MB}$ and lower EF and FS. Levosimendan is widely used as a cardioprotective positive inotropic drug, and its administration significantly improved EF, FS, cTNI, and CK-MB levels. This is consistent with previous studies of levosimendan in myocardial protection (25).

Inflammation, oxidative stress disorder, and mitochondrial dysfunction, play an important role in the pathogenesis of sepsis-induced cardiac dysfunction. Septic cardiac dysfunction is closely associated with the excessive production of pro-inflammatory cytokines such as IL$1 \beta$ and TNF- $\alpha(26,27)$. Levosimendan has been shown to have an anti-inflammatory effect (17). In our study, serum TNF- $\alpha$ and IL- $1 \beta$ elevation caused by LPS were significantly attenuated by levosimendan administration. Thus, levosimendan may have a protective effect on septic myocardial injury through anti-inflammatory mechanisms. While being good markers of oxidative stress, GSH, SOD, and MDA can act as a cofactor for a variety of enzymes, helping to repair DNA, remove $\mathrm{H}_{2} \mathrm{O}_{2}, \mathrm{HO}$, and lipid peroxides, and regenerate other antioxidants, such as ascorbic acid and tocopherols. In our study, sepsis 

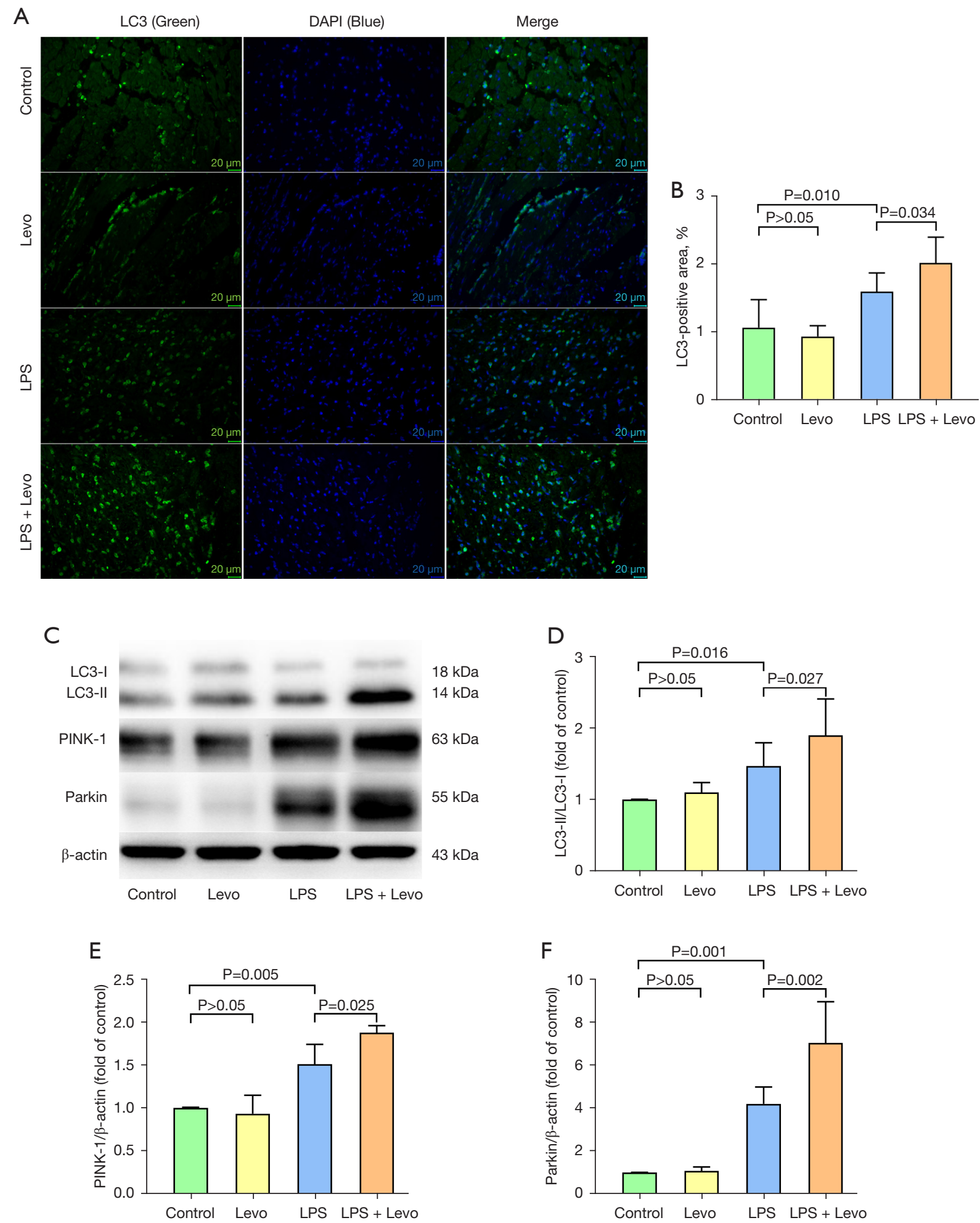

Figure 4 Levosimendan administration on myocardial mitophagy, mitophagy pathway after LPS injury. (A) Representative images LC3 dots LV tissue sections from mice in each group $(n=6)$; Nucleus is blue by labeling with DAPI, positive cells are green. (B) Quantitative analysis of LC3 in LV tissue sections from mice in each group (n=6); (C-F) representative bands of western blot and quantitative analysis of LC3B-II/LC3B-I ratio, PINK-1 expression and Parkin expression (n=6). LPS, lipopolysaccharide; Levo, Levosimendan. 
Page 10 of 16
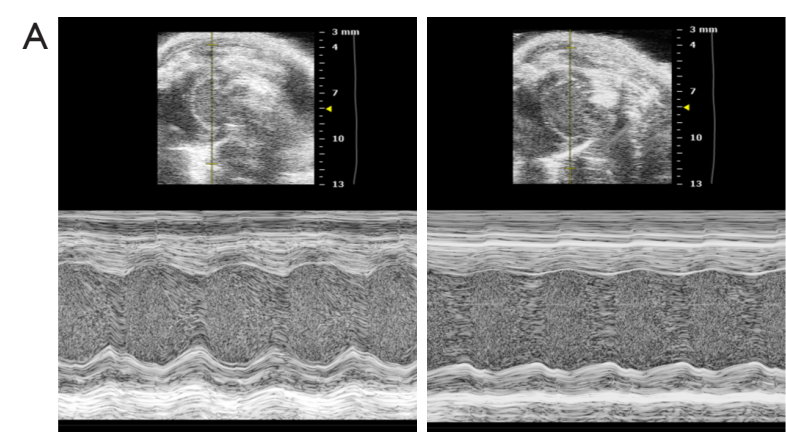

Control
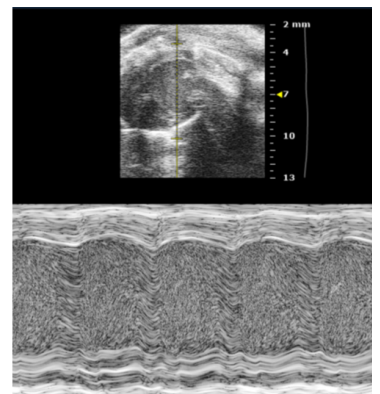

LPS + Levo

F

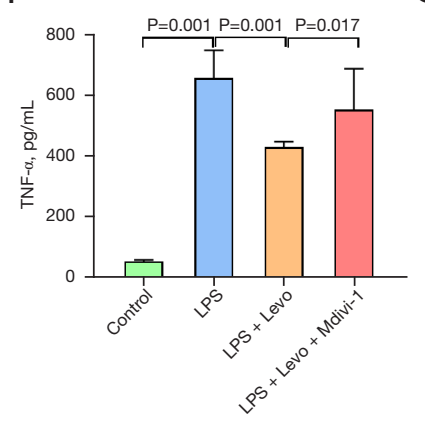

Shi et al. Levosimendan and sepsis-inducing cardiac dysfunction

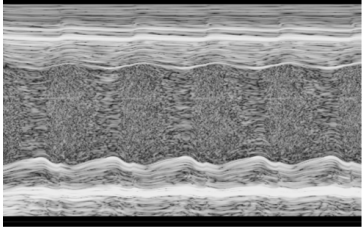

G

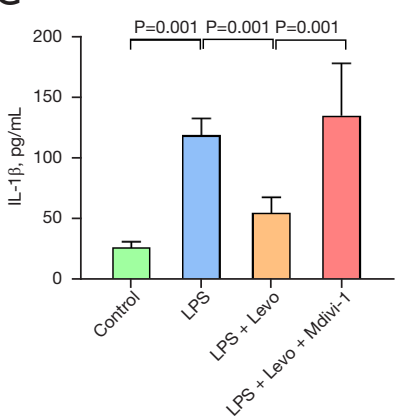

B

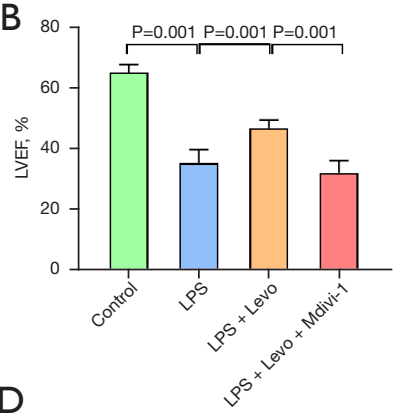

D

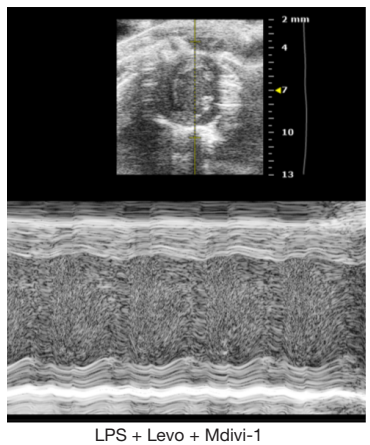

LPS + Levo + Mdivi-1

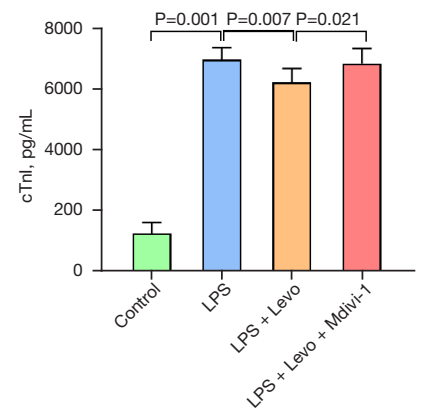

$\mathrm{H}$

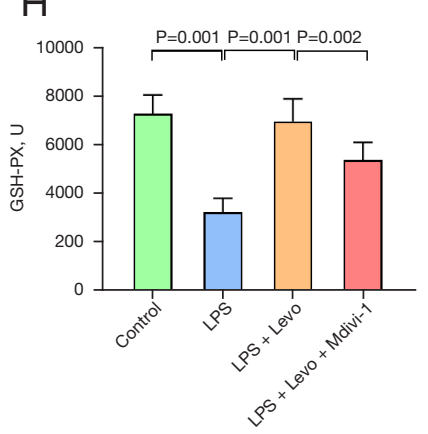

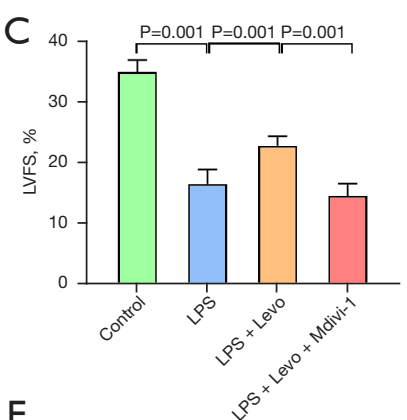

E

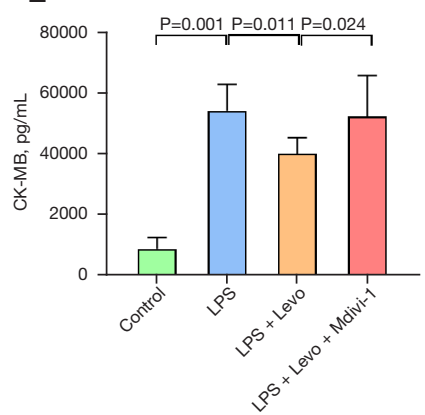

I

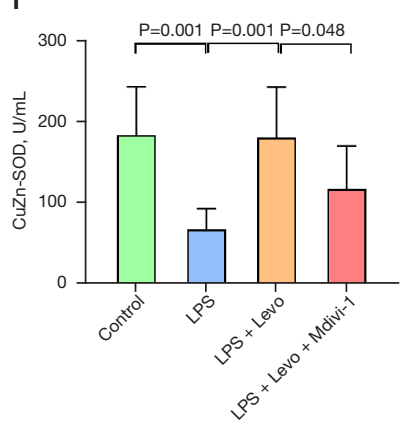



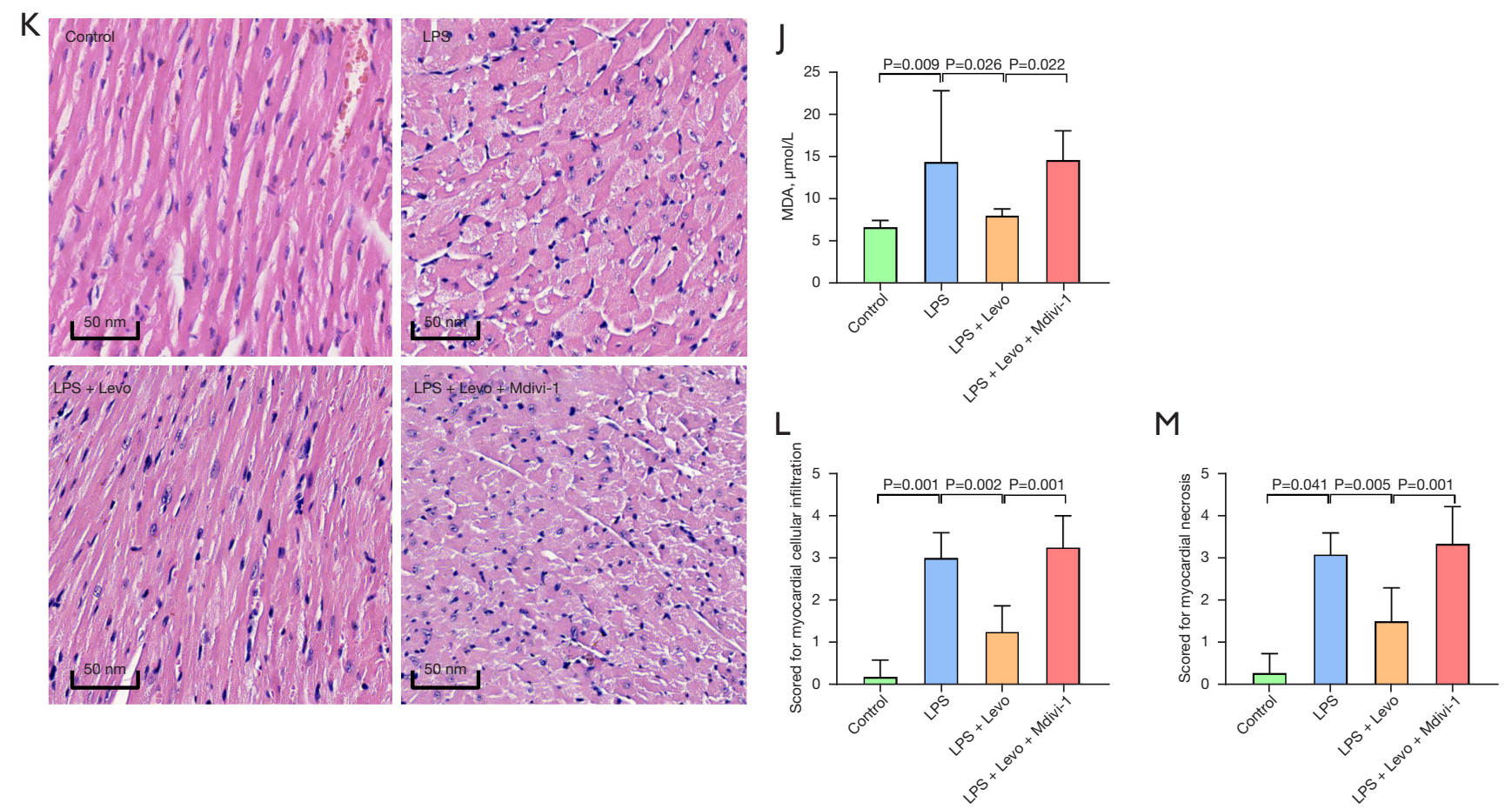

Figure 5 Mdivi-1 treatment exacerbated LPS-induced injury and impaired levosimendan-induced cardiac protection. (A-C) Representative M-mode images from mice in each group and quantitative analysis of mice LVEF and LVFS (n=6); (D,E) evaluation of myocardial injury by cTnI and CK-MB in serum (n=6); (F,G) evaluation of cardiac inflammation by TNF- $\alpha$ and IL-1 $\beta$ in serum (n=6); (H-J) evaluation of cardiac oxidative stress by GSH-Px, CuZn-SOD, and MDA in serum (n=6); (K) representative HE-stained cardiac sections (n=6); (L,M) myocardial cellular infiltration and necrosis score of 4 groups $(n=6)$. LPS, lipopolysaccharide; LVEF, left ventricular ejection fraction; LVFS, left ventricular fractional shortening; cTnI, cardiac troponin I; CK-MB, creatine kinase-myocardial isoenzyme; TNF- $\alpha$, tumor necrosis factor- $\alpha$; IL-1 $\beta$, interleukin-1 $\beta$; GSH-Px, glutathione-peroxidase; CuZn-SOD, CuZn-superoxide dismutase; MDA, malondialdehyde; Levo, Levosimendan.

reduced GSH-Px and CuZn-SOD activities and resulted in MDA elevation, leading to iliac crest loss, mitochondrial swelling, and vacuole formation, and levosimendan exerted antioxidant activity in the myocardium. Previous research has demonstrated that mitochondria limit the availability of inflammasome activators and/or components, and reduce mitochondrial DAMPs via mitophagy, and then control inflammation $(28,29)$. Furthermore, mitochondria are both the sources and targets of oxidants, and play a critical role in redox dysregulation (30). Therefore, we can speculate that levosimendan may have the effect of anti-inflammatory and antioxidant stress by protecting mitochondria.

In animal experiments and clinical studies on sepsis, cardiomyocytes manifested as mitochondrial fragmentation and mitochondrial ultrastructural damage (31). Mitochondrial fragmentation, on the other hand, relies on the activity of a cytosolic molecule, dynamic- related protein-1 (Drp-1), which is the main mediator of mitochondrial fission and encoded by Dnm11 in mice and DNM1L in humans (32). We observed that LPS-treated mice exhibited cardiac characteristics of mitochondrial fragmentation/fission, such as small-size mitochondria and phosphorylation Drp-1 (p-Drp-1), and levosimendan prevented both Drp-1 phosphorylation and mitochondrial fragmentation. Mitochondrial fission inhibition suppressed the formation of autophagosome, leading to the accumulation of damaged mitochondria and promoted the systolic dysfunction of cardiomyocytes. However, fission is not an isolated phenomenon, as it is associated with processes such as the regulation of mitophagy. In order to further investigate the mechanisms of mitochondrial dysfunction, we focused on mitophagy. In our study, LPS exposure caused rapid initiation of mitophagy, which was demonstrated by autophagosome formation and LC3II 
A
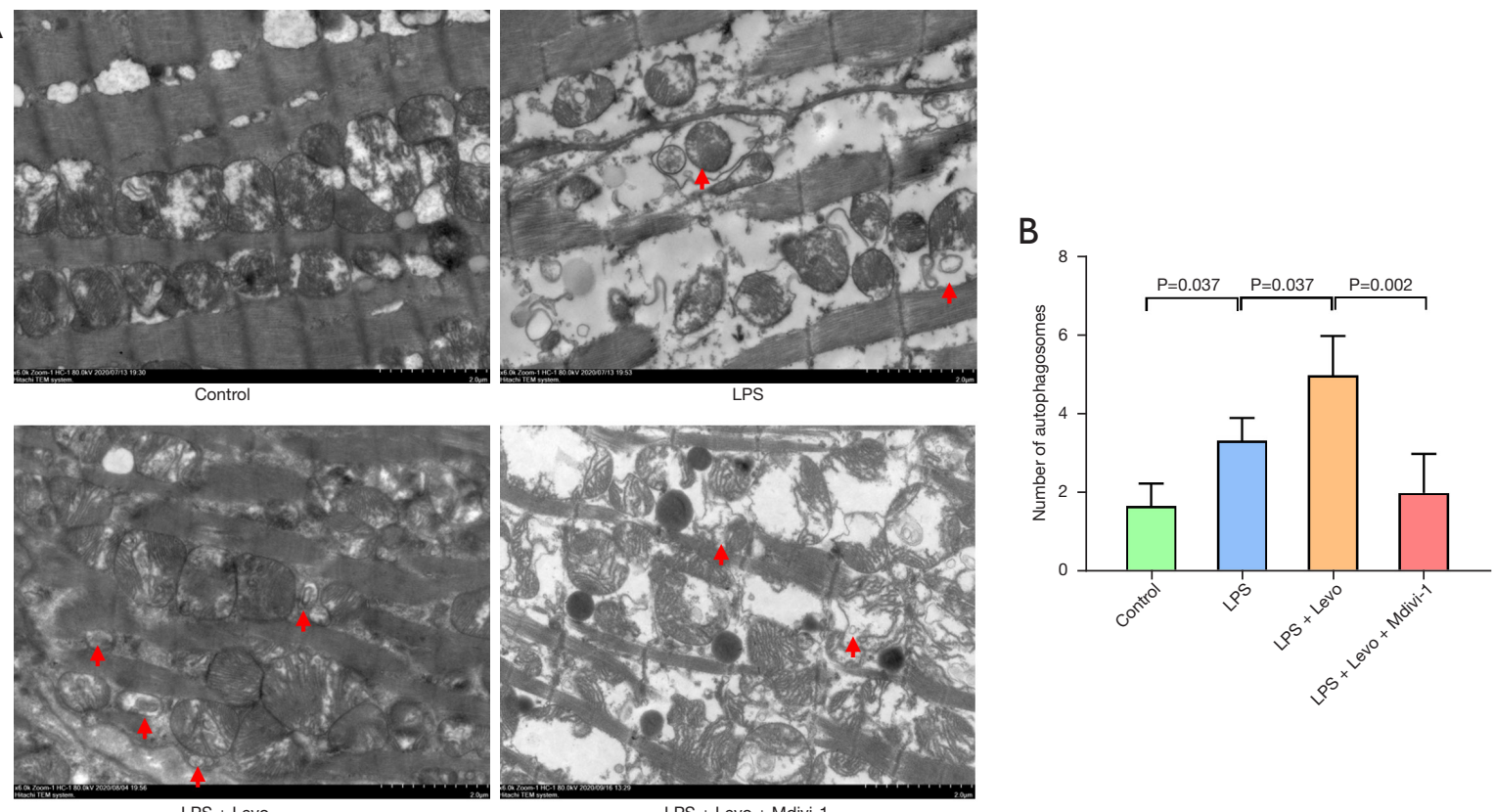

LPS + Levo

LPS + Levo + Mdivi-1
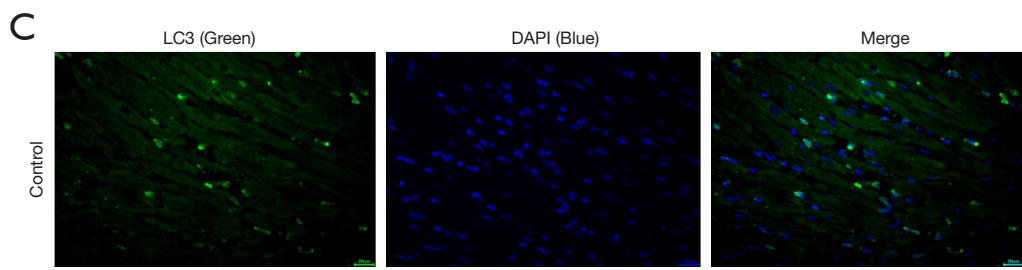

$\mathrm{D}$
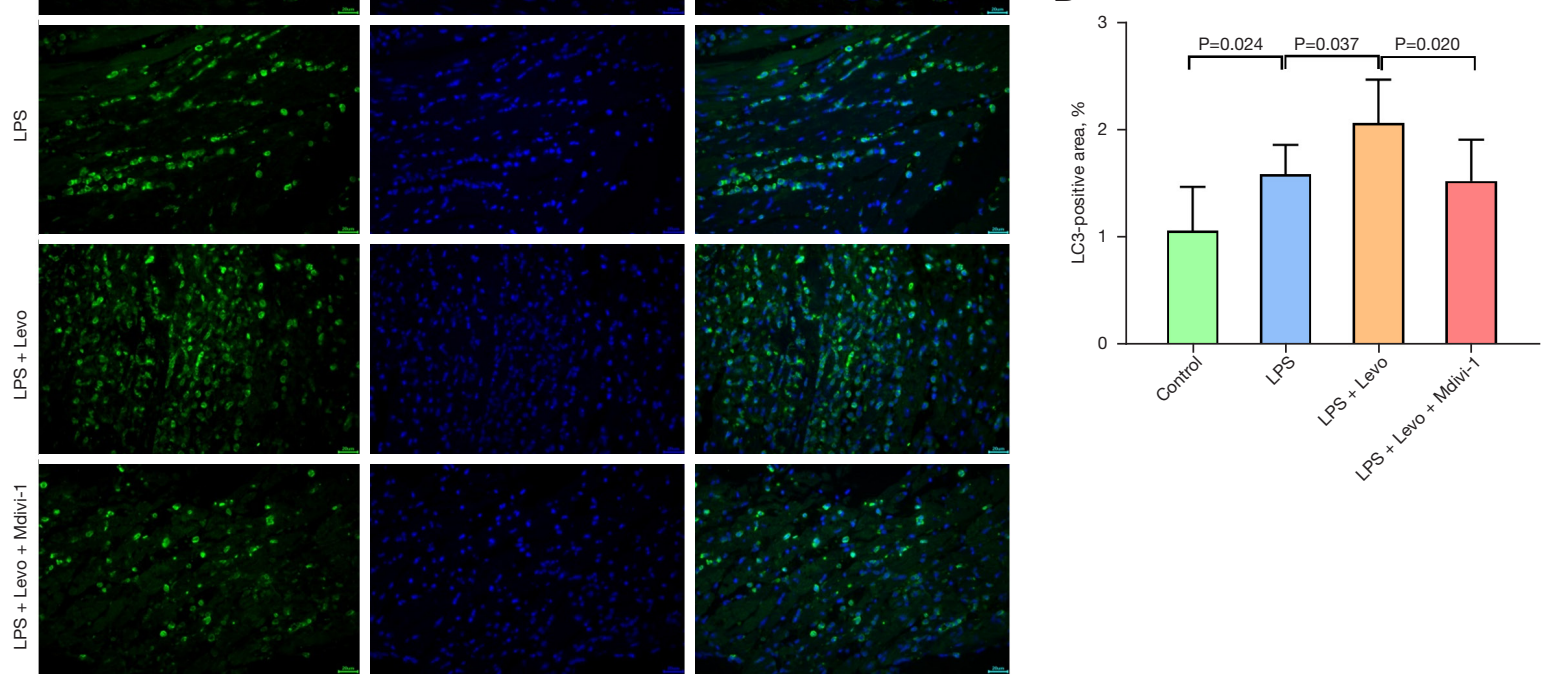

Figure 6 Mdivi-1 reversed levosimendan-induced cardiac protection via inhibiting autophagy activation. (A) Representative images of mitochondrial morphology and autophagosomes in the myocardium $(\mathrm{n}=6)$, the red arrow indicates an autophagosome; (B) quantitative analysis the number of autophagosomes in cardiomyocytes $(\mathrm{n}=6)$; (C) representative images of LC3 dots LV tissue sections from mice in each group ( $\mathrm{n}=6$ ), Nucleus is blue by labeling with DAPI, Positive cells are green; (D) quantitative analysis of LC3 in LV tissue sections from mice in each group ( $\mathrm{n}=6)$. LPS, lipopolysaccharide; Levo, Levosimendan. 
A

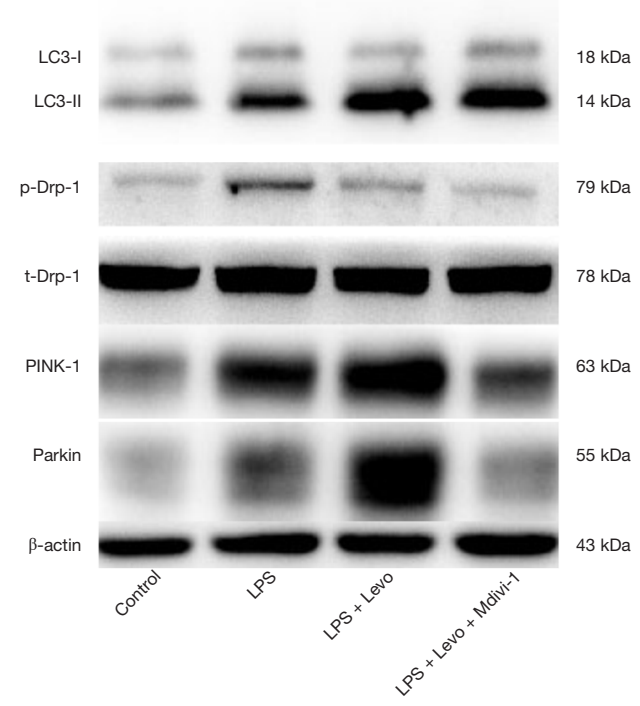

B

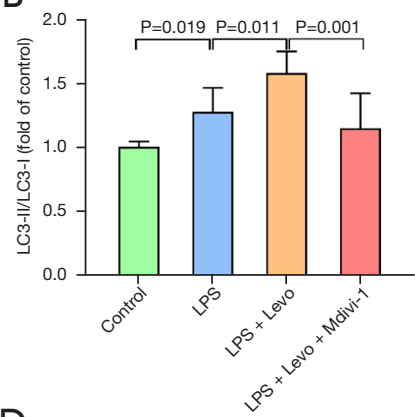

$\mathrm{D}$

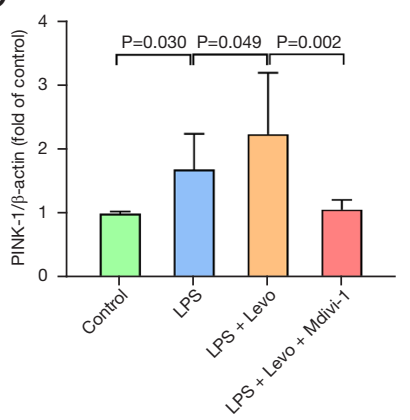

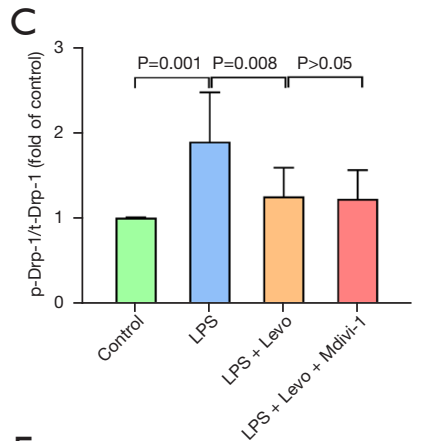

$\mathrm{E}$

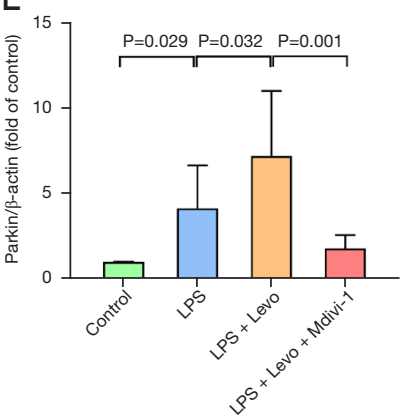

Figure 7 Mdivi-1 reversed levosimendan-induced cardiac protection via inhibiting mitophagy pathway after LPS injury. (A-E) Representative bands of western blot and quantitative analysis of p-Drp-1 phosphorylation levels, LC3B-II/LC3B-I ratio, PINK-1 expression and Parkin expression ( $\mathrm{n}=6$ ); LPS, lipopolysaccharide; Levo, Levosimendan.

conversion, and levosimendan further promoted this process. This is in consistent with previous findings in LPS and bacterial sepsis models. However, insufficient mitophagy led to mitochondrial dysfunction and adverse consequences, whereas complete induction of autophagy was essential for cardiac protection (33).

Mitochondria constantly adapt to their morphology by fusion and fission. Under the influence of a variety of stressors, mitochondria fragment into smaller entities through DRP1-mediated fission. Mitochondrial membrane potential reduction leads to stabilization of PINK1, which in turn recruits and phosphorylates the E3-ligase Parkin. Poly ubiquitinated proteins serve as a "tag" for autophagy receptors that interact with the autophagosomeresident protein LC3 (14). The central role of the PINK1Parkin pathway in the regulation of mitophagy has been demonstrated in several studies (34). Therefore, we further detected the expression of PINK-1 and Parkin, which revealed that LPS could increase the expression of PINK-1 and Parkin, and levosimendan could further enhance them. We inferred that levosimendan attenuated LPS-induced myocardial dysfunction may function through activating PINK-1-Parkin pathway.
However, it remains unclear whether the PINK-1-Parkin pathway is involved in the protective effects of levosimendan against sepsis-induced myocardial dysfunction. Our study used mdivi-1, which is a potent inhibitor of mitochondrial division dynamin-related GTPase and a specific inhibitor of the PINK-1-Parkin pathway, to verify this hypothesis and found that mdivi-1 could prevent the improvement of cardiac function such as LVEF and LVFS, myocardial enzyme markers such as cTNI and CK-MB, inflammatory cytokines levels, oxidative stress index, and myocardial pathological changes by levosimendan administration in LPS-induced cardiac dysfunction mice. Furthermore mdivi-1 markedly reduced Drp-1 phosphorylation, mitochondrial fragmentation, autophagosome formation and LC3II conversion. Defects of autophagosome formation in LPS mice treated by mdivi-1 were correlated with lysosome vacuoles increases within the myocardium, which suggest that autophagic vacuoles fail to trap mitochondria cargo (35).

In the present study, levosimendan exerted its protective effects against LPS-induced cardiac dysfunction through mitophagy and PINK-1-Parkin signaling. However, whether the other signaling pathway induces mitophagy 
has not been investigated in our article, and whether levosimendan has a protective effect on cardiac dysfunction in patients with sepsis requires further clinical trials. In addition, this model had the same limitations for example, vital signs such as hemodynamic monitoring are difficult, and there are differences between mouse models and human diseases as the typical characteristic of human sepsis infection is local infection followed by systemic infection, and it is multi bacterial infection, including gram-negative bacteria and gram-positive bacteria. In this study, there was a certain incidence rate of myocardial dysfunction due to sepsis. In order to reduce the number of mice, 6 mice were eventually analyzed by statistical analysis, and the sample size was small, which may have led to statistical deviation.

Taken together, we consider that the improved status of the mitochondria in the hearts of LPS mice is likely the result of multiple signaling regulations such as inflammation and oxidative stress. The induction of mitophagy by levosimendan preserved cardiac function and suppressed the increase in circulating cytokines following LPS challenge. Levosimendan was able to rescue the LPS-induced cardiac dysfunction mice, supporting its mechanism of action by directly targeting the PINK-1-Parkin pathway. This provides evidence for the use of levosimendan in sepsis patients with cardiac dysfunction. We will investigate these aspects in future studies to fully understand the other therapies in sepsis.

\section{Acknowledgments}

Funding: This study was supported by the Department of Science and Technology of Hebei Province of China (20277707D).

\section{Footnote}

Reporting Checklist: The authors have completed the ARRIVE reporting checklist. Available at https://atm. amegroups.com/article/view/10.21037/atm-22-483/rc

Data Sharing Statement: Available at https://atm.amegroups. com/article/view/10.21037/atm-22-483/dss

Conflicts of Interest: All authors have completed the ICMJE uniform disclosure form (available at https://atm. amegroups.com/article/view/10.21037/atm-22-483/coif). The authors have no conflicts of interest to declare.
Ethical Statement: The authors are accountable for all aspects of the work in ensuring that questions related to the accuracy or integrity of any part of the work are appropriately investigated and resolved. Experiments were performed under a project license (No. 2020002) granted by the Laboratory Animal Ethical Committee Fourth Hospital Hebei Medical University, in compliance with the National Institutes of Health (USA) Guidelines for the care and use of animals (NIH Publication No. 85-23, revised 1985: http://grants1.nih.gov/grants/olaw/references/phspol.htm)

Open Access Statement: This is an Open Access article distributed in accordance with the Creative Commons Attribution-NonCommercial-NoDerivs 4.0 International License (CC BY-NC-ND 4.0), which permits the noncommercial replication and distribution of the article with the strict proviso that no changes or edits are made and the original work is properly cited (including links to both the formal publication through the relevant DOI and the license). See: https://creativecommons.org/licenses/by-nc-nd/4.0/.

\section{References}

1. Ehrman RR, Sullivan AN, Favot MJ, et al. Pathophysiology, echocardiographic evaluation, biomarker findings, and prognostic implications of septic cardiomyopathy: a review of the literature. Crit Care 2018;22:112.

2. Stanzani G, Duchen MR, Singer M. The role of mitochondria in sepsis-induced cardiomyopathy. Biochim Biophys Acta Mol Basis Dis 2019;1865:759-73.

3. Piquereau J, Godin R, Deschênes S, et al. Protective role of PARK2/Parkin in sepsis-induced cardiac contractile and mitochondrial dysfunction. Autophagy 2013;9:1837-51.

4. Zhong J, Tan Y, Lu J, et al. Therapeutic contribution of melatonin to the treatment of septic cardiomyopathy: A novel mechanism linking Ripk3-modified mitochondrial performance and endoplasmic reticulum function. Redox Biol 2019;26:101287.

5. Tahrir FG, Langford D, Amini S, et al. Mitochondrial quality control in cardiac cells: Mechanisms and role in cardiac cell injury and disease. J Cell Physiol 2019;234:8122-33.

6. Sun Y, Cai Y, Zang QS. Cardiac Autophagy in Sepsis. Cells 2019;8:141.

7. Twig G, Elorza A, Molina AJ, et al. Fission and selective fusion govern mitochondrial segregation and elimination 
by autophagy. EMBO J 2008;27:433-46.

8. Sun Y, Yao X, Zhang QJ, et al. Beclin-1-Dependent Autophagy Protects the Heart During Sepsis. Circulation 2018;138:2247-62.

9. Vásquez TC, García CI, Pennanen C, et al. Mitochondrial dynamics, mitophagy and cardiovascular disease. J Physiol 2016;594:509-25.

10. Grossini E, Caimmi PP, Platini F, et al. Modulation of programmed forms of cell death by intracoronary levosimendan during regional myocardial ischemia in anesthetized pigs. Cardiovasc Drugs Ther 2010;24:5-15.

11. Orstavik O, Ata SH, Riise J, et al. Inhibition of phosphodiesterase- 3 by levosimendan is sufficient to account for its inotropic effect in failing human heart. Br J Pharmacol 2014;171:5169-81.

12. Liu DH, Ning YL, Lei YY, et al. Levosimendan versus dobutamine for sepsis-induced cardiac dysfunction: a systematic review and meta-analysis. Sci Rep 2021;11:20333.

13. White M, Ducharme A, Ibrahim R, et al. Increased systemic inflammation and oxidative stress in patients with worsening congestive heart failure: improvement after short-term inotropic support. Clin Sci (Lond) 2006;110:483-9.

14. Morales PE, Arias-Durán C, Ávalos-Guajardo Y, et al. Emerging role of mitophagy in cardiovascular physiology and pathology. Mol Aspects Med 2020;71:100822.

15. Schellekens WJ, van Hees HW, Linkels $M$, et al. Levosimendan affects oxidative and inflammatory pathways in the diaphragm of ventilated endotoxemic mice. Crit Care 2015;19:69.

16. Wang J, Chen H, Zhou Y, et al. Levosimendan Pretreatment Inhibits Myocardial Apoptosis in Swine after Coronary Microembolization. Cell Physiol Biochem 2017;41:67-78.

17. Wang Q, Yokoo H, Takashina M, et al. Anti-Inflammatory Profile of Levosimendan in Cecal Ligation-Induced Septic Mice and in Lipopolysaccharide-Stimulated Macrophages. Crit Care Med 2015;43:e508-20.

18. Umbarawan Y, Syamsunarno MRAA, Obinata H, et al. Robust suppression of cardiac energy catabolism with marked accumulation of energy substrates during lipopolysaccharide-induced cardiac dysfunction in mice. Metabolism 2017;77:47-57.

19. Efentakis P, Varela A, Chavdoula E, et al. Levosimendan prevents doxorubicin-induced cardiotoxicity in timeand dose-dependent manner: implications for inotropy. Cardiovasc Res 2020;116:576-91.

20. Preau S, Delguste F, Yu Y, et al. Endotoxemia Engages the RhoA Kinase Pathway to Impair Cardiac Function By Altering Cytoskeleton, Mitochondrial Fission, and Autophagy. Antioxid Redox Signal 2016;24:529-42.

21. Mao S, Chen P, Li T, et al. Tongguan Capsule Mitigates Post-myocardial Infarction Remodeling by Promoting Autophagy and Inhibiting Apoptosis: Role of Sirt1. Front Physiol 2018;9:589.

22. Kishimoto C, Kawamata H, Sakai S, et al. Enhanced production of macrophage inflammatory protein 2 (MIP-2) by in vitro and in vivo infections with encephalomyocarditis virus and modulation of myocarditis with an antibody against MIP-2. J Virol 2001;75:1294-300.

23. Vanasco V, Saez T, Magnani ND, et al. Cardiac mitochondrial biogenesis in endotoxemia is not accompanied by mitochondrial function recovery. Free Radic Biol Med 2014;77:1-9.

24. Yin X, Xin H, Mao S, et al. The Role of Autophagy in Sepsis: Protection and Injury to Organs. Front Physiol 2019;10:1071.

25. Yamashita S, Suzuki T, Iguchi K, et al. Cardioprotective and functional effects of levosimendan and milrinone in mice with cecal ligation and puncture-induced sepsis. Naunyn Schmiedebergs Arch Pharmacol 2018;391:1021-32.

26. Kumar A, Thota V, Dee L, et al. Tumor necrosis factor alpha and interleukin 1beta are responsible for in vitro myocardial cell depression induced by human septic shock serum. J Exp Med 1996;183:949-58.

27. Schumacher SM, Naga Prasad SV. Tumor Necrosis Factor- $\alpha$ in Heart Failure: an Updated Review. Curr Cardiol Rep 2018;20:117.

28. Shi CS, Shenderov K, Huang NN, et al. Activation of autophagy by inflammatory signals limits IL- $1 \beta$ production by targeting ubiquitinated inflammasomes for destruction. Nat Immunol 2012;13:255-63.

29. Oka T, Hikoso S, Yamaguchi O, et al. Mitochondrial DNA that escapes from autophagy causes inflammation and heart failure. Nature 2012;485:251-5.

30. Mantzarlis K, Tsolaki V, Zakynthinos E. Role of Oxidative Stress and Mitochondrial Dysfunction in Sepsis and Potential Therapies. Oxid Med Cell Longev 2017;2017:5985209.

31. Soriano FG, Nogueira AC, Caldini EG, et al. Potential role of poly(adenosine 5'-diphosphate-ribose) polymerase activation in the pathogenesis of myocardial contractile dysfunction associated with human septic shock. Crit Care Med 2006;34:1073-9.

32. Pitts KR, Yoon Y, Krueger EW, et al. The dynamin- 
like protein DLP1 is essential for normal distribution and morphology of the endoplasmic reticulum and mitochondria in mammalian cells. Mol Biol Cell 1999;10:4403-17.

33. Hsieh CH, Pai PY, Hsueh HW, et al. Complete induction of autophagy is essential for cardioprotection in sepsis. Ann Surg 2011;253:1190-200.

34. Matsuda N, Sato S, Shiba K, et al. PINK1 stabilized by

Cite this article as: Shi J, Chen Y, Zhi H, An H, Hu Z. Levosimendan protects from sepsis-inducing cardiac dysfunction by suppressing inflammation, oxidative stress and regulating cardiac mitophagy via the PINK-1-Parkin pathway in mice. Ann Transl Med 2022;10(4):212. doi: 10.21037/atm$22-483$ mitochondrial depolarization recruits Parkin to damaged mitochondria and activates latent Parkin for mitophagy. J Cell Biol 2010;189:211-21.

35. Chen S, Zhou L, Zhang Y, et al. Targeting SQSTM1/p62 induces cargo loading failure and converts autophagy to apoptosis via NBK/Bik. Mol Cell Biol 2014;34:3435-49.

(English Language Editor: J. Jones) 